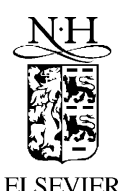

\title{
The dynamics of the Goldstone bosons on the brane
}

\author{
Antonio Dobado $^{\mathrm{a}, *}$, Antonio L. Maroto ${ }^{\mathrm{a}, \mathrm{b}}$ \\ a Departamento de Física Teórica, Universidad Complutense de Madrid, 28040 Madrid, Spain \\ b CERN Theory Division, CH-1211 Geneva 23, Switzerland
}

Received 17 July 2000; accepted 20 September 2000

\begin{abstract}
We study, within the recently proposed brane-world scenario, the effective action for the lowenergy brane excitations. These modes are understood as Goldstone bosons associated to the spontaneous isometry breaking, induced on the bulk space by the presence of the brane. Starting from the Nambu-Goto action for the brane, we obtain a non-linear sigma model describing the low-energy interactions of the Goldstone bosons and extend the calculation up to $\mathcal{O}\left(p^{4}\right)$. We also discuss the Higgs-like mechanism in which the Kaluza-Klein gauge fields absorb the Goldstone bosons and acquire mass. Finally, we present the explicit form of the effective action describing the low-energy interactions between the three-brane Goldstone bosons and the particles present in the Standard Model. @ 2001 Elsevier Science B.V. All rights reserved.
\end{abstract}

PACS: 11.25.Mj; 11.10.Lm; 11.15.Ex

\section{Introduction}

The existence of large extra dimensions has been proposed [1] as a possible explanation of the enormous difference between the electroweak $\left(M_{W}\right)$ and gravitational $\left(M_{P}\right)$ scales, which typically is of the order of $M_{P} / M_{W} \simeq 10^{16}$. In this proposal, the most relevant scale would be the Planck scale in $D=4+n$ dimensions $M_{D}$, which is assumed to be not too far from the $\mathrm{TeV}$ scale in order to solve the above-mentioned hierarchy problem. The much larger value of the four-dimensional Planck constant would be due to the size of the extra dimensions, since we typically have $M_{P}^{2} \simeq R^{n} M_{D}^{n+2}$, where $R$ is the radius of the compactified extra dimensions. Phenomenological considerations then require that, whereas gravity can propagate in the $D$-dimensional bulk space, the ordinary matter fields and gauge bosons be bound to live on a three-dimensional brane, which would constitute

\footnotetext{
* Corresponding author. Phone: +34 9139451 92; Fax: +34 913945197.

E-mail address: dobado@eucmax.sim.ucm.es (A. Dobado).
} 
the usual spatial dimensions. The brane tension $f$ is the other important scale in this scenario, $f^{-1}$ being the typical size of the brane fluctuations.

A large number of works have been devoted to the study of the phenomenological implications of this scenario (see [2-4] and references therein). In particular, special attention has been paid to the description of the low-energy sector of the model. This sector would include the Standard Model (SM) fields, the gravitons and the possible excitations of the brane [5]. The presence of extra dimensions allows for the existence, in addition to the standard massless gravitons in four dimensions, of an infinite tower of massive gravitons (Kaluza-Klein [6,7] gravitons) whose mass is determined by the size of the extra dimensions (see, for example, [8] for a review of different Kaluza-Klein models). The interaction of the graviton sector with the SM fields has been analysed in a series of papers $[9,10]$ and different predictions have been obtained that could be tested at future particle colliders. However, in addition to the gravitons, the low-energy spectrum of the theory also contains the brane's own excitations. If the brane has been spontaneously created with a given shape in the bulk (that we will consider as its ground state), the initial isometries of the bulk space could be broken spontaneously by the presence of the brane. The brane configurations obtained by means of some isometry transformations in the bulk will be considered as equivalent ground states and therefore the parameters describing such transformations can be considered as zero-mode excitations of the ground state. When such transformations are made local (depending on the position on the brane), the corresponding parameters play the role of Goldstone bosons (GB) fields of the isometry breaking. Moreover, it has been shown that, in the case where the brane tension $f$ is much smaller than the fundamental scale $M_{D}\left(f \ll M_{D}\right)$, the non-zero KK modes decouple from the GB modes [11-13] and it is then possible, at least in principle, to make a lowenergy effective theory description of the GB dynamics. On the other hand, in the standard Kaluza-Klein models, the isometries in the extra dimensions are understood as gauge transformations in the four-dimensional theory. Therefore, since the GB are associated to the breaking of those gauge transformations, it is natural to expect [2-4] that some kind of Higgs mechanism can take place, which would give mass to the Kaluza-Klein gauge bosons.

In this paper we study the low-energy dynamics of these GB and the Higgs-like mechanism we have just commented on. In Section 2 we set the main assumptions and the notation used in the rest of the work. Starting from a Nambu-Goto like action for the brane, we obtain the non-linear sigma model describing the low-energy dynamics of the brane GB. Those GB correspond to the spontaneous symmetry breaking of the translational isometries of the compactified extra dimensions by the brane. When the isometries are not exact but only approximate, the GB become pseudo-GB and acquire some mass. Section 3 is devoted to the calculation of these masses. There we compute also the next to leading order corrections to the non-linear sigma model containing four derivatives of the GB fields. In Section 4 we describe in detail how the GB can be absorbed by the KK graviphotons, giving rise to the Higgs mechanism. In Section 5 we study the couplings of GB with the fields present in the SM, including scalars, (chiral) fermions and gauge bosons. Finally, Section 6 contains the main conclusions of the work. 


\section{The effective action for the Goldstone bosons}

In the following we will consider the four-dimensional space-time $M_{4}$ to be embedded in a $D$-dimensional bulk space that for simplicity we will assume to be of the form $M_{D}=M_{4} \times B$, where $B$ is a given compact manifold. This kind of spaces include, as a particular case, the extra-dimensional tori spaces usually considered in the literature [13]. The coordinates parametrizing the points in $M_{D}$ will be denoted by $\left(x^{\mu}, y^{m}\right)$, where the different indices run as $\mu=0,1,2,3$ and $m=4,5, \ldots, D-1$. The bulk space $M_{D}$ is endowed with a metric tensor that we will denote by $G_{M N}$, with signature $(+,-,-, \cdots,-,-)$. In the absence of the 3-brane, this metric possesses an isometry group that we will assume to be of the form $G\left(M_{D}\right)=G\left(M_{4}\right) \times G(B)$. The presence of the brane spontaneously breaks this symmetry down to some subgroup $G\left(M_{4}\right) \times H$. Therefore, we can introduce the coset space $K=G\left(M_{D}\right) /\left(G\left(M_{4}\right) \times H\right)=G(B) / H$, where $H \subset G$ is a certain subgroup of $G$ that we will study below.

Let us first consider the simplest case, in which the internal manifold is just the circle $B=S^{1}$, i.e., $M_{5}=M_{4} \times S^{1}$ and the corresponding isometry group is just Abelian $G(B)=$ $U(1)$. In this case, the brane parametrization reads $Y^{M}=\left(x^{\mu}, Y(x)\right)$ and the metric tensor induced on the brane $g_{\mu \nu}$ is given by:

$$
g_{\mu \nu}=\partial_{\mu} Y^{M} \partial_{\nu} Y^{N} G_{M N} \text {. }
$$

We consider the simplest form for the metric on the bulk:

$$
G_{M N}=\left(\begin{array}{cc}
\tilde{g}_{\mu \nu}(x) & 0 \\
0 & -1
\end{array}\right) \text {. }
$$

According to (1), the induced metric on the brane is given in this case by:

$$
g_{\mu \nu}=\tilde{g}_{\mu \nu}-\partial_{\mu} Y \partial_{\nu} Y
$$

so that

$$
\sqrt{g}=(-\operatorname{det} g)^{1 / 2}=\sqrt{\tilde{g}}\left(1-\frac{1}{2} \tilde{g}^{\mu \nu} \partial_{\mu} Y \partial_{\nu} Y+\cdots\right) .
$$

Under very general assumptions, the low-energy action for a narrow 3-brane can be taken as a Nambu-Goto action (originally introduced in the context of membranes by Dirac [14]), i.e.,

$$
S_{B}=-f^{4} \int_{M_{4}} d^{4} x \sqrt{g},
$$

where $d^{4} x \sqrt{g}$ is the volume element of the brane and $f$ is the brane tension. Thus for small excitations, the effective action becomes

$$
S_{B}=-f^{4} \int_{M_{4}} d^{4} x \sqrt{\tilde{g}}+\frac{f^{4}}{2} \int_{M_{4}} d^{4} x \sqrt{\tilde{g}} \tilde{g}^{\mu \nu} \partial_{\mu} Y \partial_{\nu} Y .
$$

In this simple case there is only one Goldstone boson that parametrizes the small excitations of the brane as $Y(x)$, and can be identified with the coordinate in the internal dimension. As we will see this is not always the case for more general spaces $B$. 
Let us thus consider internal spaces with higher dimensions. Then the isometry group is in general non-Abelian. We introduce the $T_{a}(a=1, \ldots, \operatorname{dim}(G(B)))$ which are the generators of $G(B)$ with commutation relations:

$$
\left[T_{a}, T_{b}\right]=i C_{a b c} T_{c},
$$

where $C_{a b c}$ are the structure constants. The $B$ Killing vectors $\xi_{a}(y)$ satisfy the $G(B)$ Lie algebra:

$$
\left\{\xi_{a}, \xi_{b}\right\}=i C_{a b c} \xi_{c}
$$

where the brackets are the standard Lie brackets. The brane parametrization in this case can be written as $Y^{M}=\left(x^{\mu}, Y^{m}(x)\right)$. The brane is created with a shape $Y^{m}(x)$ that minimizes the action (4). In particular a possible solution corresponds to $Y^{m}(x)=Y_{0}^{m}$, i.e., the brane is created in a certain point in $B$. In this case the presence of the brane will break spontaneously all the $B$ isometries, except for those that leave the point $Y_{0}$ unchanged. In other words the group $G(B)$ is spontaneously broken down to $H\left(Y_{0}\right)$, where $H\left(Y_{0}\right)$ denotes the isotropy group (or little group) of the point $Y_{0}$. Let $H_{i}$ be the $H$ generators $(i=1,2, \ldots, h), X_{\alpha}(\alpha=1,2, \ldots, k=\operatorname{dim} G-\operatorname{dim} H)$ the broken generators, and $T=(H, X)$ the complete set of generators of $G$. A similar separation can be done with the Killing fields. We will denote $\xi_{i}$ those associated to the $H_{i}$ generators and $\xi_{\alpha}$ those corresponding to $X_{\alpha}$. The excitations of the brane along the (broken) Killing fields directions of $B$ correspond to the zero modes and they are parametrized by the GB fields $\pi^{\alpha}(x)$ that can be understood as coordinates on the coset manifold $K=G / H$. Thus, let us assume again that the brane ground state is position-independent $Y_{0}^{m}$, then the action of an element of $G(B)$ on $B$ will map $Y_{0}$ into some other point with coordinates:

$$
Y^{m}(x)=Y^{m}\left(Y_{0}, \pi^{\alpha}(x)\right)=Y_{0}^{m}+\frac{1}{k f^{2}} \xi_{\alpha}^{m}\left(Y_{0}\right) \pi^{\alpha}(x)+\mathcal{O}\left(\pi^{2}\right),
$$

where we have set the appropriate normalization of the GB fields and Killing fields with $k^{2}=16 \pi / M_{P}^{2}$. It is important to note that the coordinates of the transformed point depend only on $\pi^{\alpha}(x)$, i.e., on the parameters of the transformations corresponding to the broken generators. The rest of the transformations (corresponding to the $H$ subgroup) leave the vacuum unchanged and therefore they are not GB. Thus not all the isometries will give rise to zero modes of the brane.

When the action of the isometry group $G(B)$ on $B$ is transitive, i.e., when any pair of points in $B$ can be connected by an isometry transformation, $B$ is said to be a homogeneous space. In this case, the isotropy group of a given point is independent of the particular point we have chosen, i.e., $H\left(Y_{0}\right)=H$, and, under certain regularity conditions [15], it is possible to prove that $B$ is diffeomorphic to $K=G / H$. As a consequence, in those cases, the number of GB coincides with the number of extra dimensions ( $\operatorname{dim} B=\operatorname{dim} K=$ $\operatorname{dim} G-\operatorname{dim} H$ ). Typical examples of this case are $B=T^{n}, S^{n}$ or $S U(N)$. However, if $B$ is not a homogenous space, this result does not hold and the number of GB (which equals $\operatorname{dim} K$ ) will be in general smaller than $\operatorname{dim} B$. Thus, for example, consider $B$ to be a twodimensional ellipsoid with axial symmetry. In this case, $G(B)=U(1)$ and $\operatorname{dim} G(B)=1$. The isotropy group now depends on the particular point we choose. If $Y_{0}$ corresponds to 
any of the two poles on the symmetry axis, then $H\left(Y_{0}\right)=U(1)$, i.e., $\operatorname{dim} H\left(Y_{0}\right)=1$ and the number of GB is zero. However, for a generic point, $H\left(Y_{0}\right)=1$ or $H\left(Y_{0}\right)=Z_{2}$ for points on the equator, therefore $\operatorname{dim} H\left(Y_{0}\right)=0$ and the number of GB is one. In any case $\operatorname{dim} K \neq \operatorname{dim} B$.

In order to find the GB effective action for arbitrary dimensions, we consider the bulk metric ansatz as:

$$
G_{M N}=\left(\begin{array}{cc}
\tilde{g}_{\mu \nu}(x) & 0 \\
0 & -g_{m n}^{\prime}(y)
\end{array}\right) \text {. }
$$

In the ground state, the induced metric on the brane is given by the four-dimensional components of the bulk space metric, i.e., $g_{\mu \nu}=\tilde{g}_{\mu \nu}=G_{\mu \nu}$. When brane excitations are present, the induced metric is given by $g_{\mu \nu}=\tilde{g}_{\mu \nu}-\partial_{\mu} Y^{m} \partial_{\nu} Y^{n} g_{m n}^{\prime}$, where

$$
\partial_{\mu} Y^{m}(x)=\frac{\partial Y^{m}}{\partial \pi^{\alpha}} \partial_{\mu} \pi^{\alpha}=\frac{1}{k f^{2}} \xi_{\alpha}^{m}\left(Y_{0}\right) \partial_{\mu} \pi^{\alpha}+\mathcal{O}\left(\pi^{2}\right),
$$

and, accordingly:

$$
g_{\mu \nu}=\tilde{g}_{\mu \nu}-g_{m n}^{\prime} \frac{\partial Y^{m}}{\partial \pi^{\alpha}} \frac{\partial Y^{n}}{\partial \pi^{\beta}} \partial_{\mu} \pi^{\alpha} \partial_{\nu} \pi^{\beta} .
$$

Introducing the tensor $h_{\alpha \beta}(\pi)$ as

$$
h_{\alpha \beta}(\pi)=f^{4} g_{m n}^{\prime}(Y(\pi)) \frac{\partial Y^{m}}{\partial \pi^{\alpha}} \frac{\partial Y^{n}}{\partial \pi^{\beta}},
$$

we have

$$
g_{\mu \nu}=\tilde{g}_{\mu \nu}-\frac{1}{f^{4}} h_{\alpha \beta}(\pi) \partial_{\mu} \pi^{\alpha} \partial_{\nu} \pi^{\beta},
$$

and, for the square root of the metric determinant, we get

$$
\sqrt{g}=\sqrt{\tilde{g}}\left(1-\frac{1}{2 f^{4}} \tilde{g}^{\mu \nu} h_{\alpha \beta}(\pi) \partial_{\mu} \pi^{\alpha} \partial_{\nu} \pi^{\beta}+\cdots\right),
$$

so that the effective action for the Goldstone bosons up to $\mathcal{O}\left(p^{2}\right)$ is nothing but the nonlinear sigma model corresponding to a symmetry-breaking pattern $G \rightarrow H$ :

$$
S_{B}^{(2)}=-f^{4} \int_{M_{4}} d^{4} x \sqrt{\tilde{g}}+\frac{1}{2} \int_{M_{4}} d^{4} x \sqrt{\tilde{g}} \tilde{g}^{\mu \nu} h_{\alpha \beta}(\pi) \partial_{\mu} \pi^{\alpha} \partial_{\nu} \pi^{\beta} .
$$

Notice that $\mu, v, \ldots$ are $M_{4}$ indices, whereas $\alpha, \beta, \ldots$ are indices on the $K$ manifold. The above expansion of the determinant in (13) and therefore that of the effective action in (14) is not an expansion in powers of the $\pi$ fields, but in powers of $\partial \pi / f^{2}$, i.e., they are low-energy expansions.

\section{Mass terms and higher order corrections}

When the $G\left(M_{D}\right)$ symmetry is not exact, it can only be written as the product $G\left(M_{4}\right) \times$ $G(B)$ in an approximate way. In this case, the Goldstone bosons are not massless, and 
their masses measure how accurately $G\left(M_{D}\right)$ describes the real symmetries of the bulk space-time. This is similar to the $S U(2)_{L} \times S U(2)_{R}$ chiral symmetries of the low-energy strong interactions. This symmetry is spontaneously broken down to the isospin symmetry $S U(2)_{L+R}$. The corresponding Goldstone bosons are the three pions. However, the small quark masses break explicitly the chiral symmetry and the above breaking pattern is only approximate. The pions then get a mass that is smaller than the rest of the hadron masses in the QCD spectrum.

When the $G\left(M_{D}\right)$ symmetry is not exact, it is no longer possible to assume that $\tilde{g}_{\mu \nu}$ depends only on $x$ and $g_{m n}^{\prime}$ on $y$. Thus, we can assume a slight dependence of $\tilde{g}_{\mu \nu}$ also on the $y$ coordinates. We will use the following ansatz in the Abelian case:

$$
G_{M N}=\left(\begin{array}{cc}
\tilde{g}_{\mu \nu}(x, y) & 0 \\
0 & -1
\end{array}\right) .
$$

It is now possible to expand $\tilde{g}_{\mu \nu}(x, y)$ around $y=Y_{0}$, where we choose the coordinates so that $y=Y_{0}$ corresponds to the appropriate vacuum when the $G\left(M_{D}\right)$ symmetry is not exact:

$$
\begin{aligned}
\tilde{g}_{\mu \nu}(x, Y)= & \tilde{g}_{\mu \nu}\left(x, Y_{0}\right)+\partial_{Y} \tilde{g}_{\mu \nu}\left(x, Y_{0}\right)\left(Y-Y_{0}\right) \\
& +\frac{1}{2} \partial_{Y}^{2} \tilde{g}_{\mu \nu}\left(x, Y_{0}\right)\left(Y-Y_{0}\right)^{2}+\cdots .
\end{aligned}
$$

Therefore, at the lowest non-trivial order we have

$$
\sqrt{g}=\sqrt{\tilde{g}}\left(1-\frac{1}{2} \tilde{g}^{\mu \nu} \partial_{\mu} Y \partial_{\nu} Y+\frac{1}{4} \tilde{g}^{\mu \nu}\left(\partial_{Y}^{2} \tilde{g}_{\mu \nu}\right) Y^{2}+\cdots\right) .
$$

Then, in terms of the properly normalized Goldstone boson field $\pi=f^{2} Y$, the low-energy effective action can be written as

$$
\begin{aligned}
S_{B}= & -f^{4} \int_{M_{4}} d^{4} x \sqrt{\tilde{g}}+\frac{1}{2} \int_{M_{4}} d^{4} x \sqrt{\tilde{g}} \tilde{g}^{\mu \nu} \partial_{\mu} \pi \partial_{\nu} \pi \\
& -\frac{1}{4} \int_{M_{4}} d^{4} x \sqrt{\tilde{g}} \tilde{g}^{\mu \nu}\left(\partial_{Y}^{2} \tilde{g}_{\mu \nu}\right) \pi^{2}+\cdots .
\end{aligned}
$$

Thus the pseudo-Goldstone boson field $\pi$ gets a mass

$$
M^{2}=\frac{1}{2} \tilde{g}^{\mu \nu}\left(\partial_{Y}^{2} \tilde{g}_{\mu \nu}\right)
$$

The general case can be studied in a similar way. We start from the ansatz

$$
G_{M N}=\left(\begin{array}{cc}
\tilde{g}_{\mu \nu}(x, y) & 0 \\
0 & -g_{m n}^{\prime}(y)
\end{array}\right),
$$

where again $\tilde{g}_{\mu \nu}\left(x, Y_{0}\right)$ corresponds to the ground state metric in the symmetric case. Expanding this metric around $y^{m}=Y_{0}^{m}$ in terms of the $\pi^{\alpha}$ fields we find

$$
\begin{aligned}
\tilde{g}_{\mu \nu}(x, y)= & \tilde{g}_{\mu \nu}\left(x, Y_{0}\right)+\partial_{m} \tilde{g}_{\mu \nu}\left(x, Y_{0}\right)\left(Y^{m}-Y_{0}^{m}\right) \\
& +\frac{1}{2} \partial_{m} \partial_{n} \tilde{g}_{\mu \nu}\left(x, Y_{0}\right)\left(Y^{m}-Y_{0}^{m}\right)\left(Y^{n}-Y_{0}^{n}\right)+\cdots
\end{aligned}
$$




$$
\begin{aligned}
= & \tilde{g}_{\mu \nu}\left(x, Y_{0}\right)+\partial_{m} \tilde{g}_{\mu \nu}\left(x, Y_{0}\right)\left(\frac{\xi_{\alpha}^{m}}{k f^{2}} \pi^{\alpha}+\left.\frac{1}{2} \frac{\partial^{2} Y^{m}}{\partial \pi^{\alpha} \partial \pi^{\beta}}\right|_{\pi=0} \pi^{\alpha} \pi^{\beta}\right) \\
& +\frac{1}{2}\left(\partial_{m} \partial_{n} \tilde{g}_{\mu \nu}\left(x, Y_{0}\right)\right) \frac{\xi_{\alpha}^{m} \xi_{\beta}^{n}}{k^{2} f^{4}} \pi^{\alpha} \pi^{\beta}+\mathcal{O}\left(\pi^{3}\right) .
\end{aligned}
$$

The effective action is then given by

$$
\begin{aligned}
S_{B}= & -f^{4} \int_{M_{4}} d^{4} x \sqrt{\tilde{g}} \\
& +\frac{1}{2} \int_{M_{4}} d^{4} x \sqrt{\tilde{g}}\left(\tilde{g}^{\mu \nu} h_{\alpha \beta}(\pi) \partial_{\mu} \pi^{\alpha} \partial_{\nu} \pi^{\beta}-M_{\alpha \beta}^{2} \pi^{\alpha} \pi^{\beta}\right)+\cdots,
\end{aligned}
$$

where $\tilde{g}^{\mu \nu}$ denotes $\tilde{g}^{\mu \nu}\left(x, Y_{0}\right)$ and the mass matrix can be written as

$$
M_{\alpha \beta}^{2}=\frac{f^{4}}{2} \tilde{g}^{\mu \nu}\left(\left.\frac{\partial^{2} Y^{m}}{\partial \pi^{\alpha} \partial \pi^{\beta}}\right|_{\pi=0} \partial_{m} \tilde{g}_{\mu \nu}+\partial_{m} \partial_{n} \tilde{g}_{\mu \nu} \frac{\xi_{\alpha}^{m} \xi_{\beta}^{n}}{k^{2} f^{4}}\right) .
$$

From the above discussion we arrive at the conclusion that the pseudo-GB masses are of the order of magnitude of the derivatives of the physical four-dimensional metric, which are expected to be of the order of the inverse brane size fluctuations $f^{-1}$, i.e., we expect the pseudo-GB masses to be of the same order of magnitude as the brane tension $f$.

Finally we will extract the $\mathcal{O}\left(p^{4}\right)$ corrections to the GB effective action. With that purpose we further expand the metric determinant $\sqrt{g}$ in terms of the metric $\tilde{g}_{\mu \nu}$ and of the GB modes derivatives $\partial \pi^{\alpha}$, following steps similar to what was done in (13). Thus we find:

$$
\begin{aligned}
\sqrt{g}=\sqrt{\tilde{g}}\left(1-\frac{1}{2 f^{4}} \tilde{g}^{\mu \nu} h_{\alpha \beta}(\pi) \partial_{\mu} \pi^{\alpha} \partial_{\nu} \pi^{\beta}\right. \\
\quad+\frac{1}{8 f^{4}} \tilde{g}^{\mu \nu} \tilde{g}^{\rho \sigma} h_{\alpha \beta}(\pi) h_{\gamma \delta}(\pi) \partial_{\mu} \pi^{\alpha} \partial_{\nu} \pi^{\beta} \partial_{\rho} \pi^{\gamma} \partial_{\sigma} \pi^{\delta} \\
\left.\quad-\frac{1}{4 f^{4}} \tilde{g}^{\mu \nu} \tilde{g}^{\rho \sigma} h_{\alpha \beta}(\pi) h_{\gamma \delta}(\pi) \partial_{\nu} \pi^{\alpha} \partial_{\rho} \pi^{\beta} \partial_{\sigma} \pi^{\gamma} \partial_{\mu} \pi^{\delta}+\cdots\right),
\end{aligned}
$$

so that the $\mathcal{O}\left(p^{4}\right)$ effective action becomes

$$
\begin{aligned}
S_{B}^{(4)}= & -f^{4} \int_{M_{4}} d^{4} x \sqrt{\tilde{g}}+\frac{1}{2} \int_{M_{4}} d^{4} x \sqrt{\tilde{g}} \tilde{g}^{\mu \nu} h_{\alpha \beta}(\pi) \partial_{\mu} \pi^{\alpha} \partial_{\nu} \pi^{\beta} \\
& -\frac{1}{8 f^{4}} \int_{M_{4}} d^{4} x \sqrt{\tilde{g}}\left(\tilde{g}^{\mu \nu} h_{\alpha \beta}(\pi) \partial_{\mu} \pi^{\alpha} \partial_{\nu} \pi^{\beta}\right)^{2} \\
& +\frac{1}{4 f^{4}} \int_{M_{4}} d^{4} x \sqrt{\tilde{g}} \tilde{g}^{\mu \nu} \tilde{g}^{\rho \sigma} h_{\alpha \beta}(\pi) h_{\gamma \delta}(\pi) \partial_{\nu} \pi^{\alpha} \partial_{\rho} \pi^{\beta} \partial_{\sigma} \pi^{\gamma} \partial_{\mu} \pi^{\delta} .
\end{aligned}
$$

The GB self-interactions are organized in increasing number of GB field derivatives over $f$. We thus have a well-defined energy expansion so that, at low energies with respect to $f$, only the first terms contribute, in much the same way as it happens in the 
Chiral Perturbation Theory $(\chi \mathrm{PT})$ which is used to describe the low-energy interactions of pions or the Electroweak Chiral Lagrangians used to describe the symmetry breaking sector of the SM (see, for instance, [16] and references therein). This kind of description is completely determined (at the lowest order) by just the symmetry-breaking pattern $K=$ $G / H$ and an energy scale, $f$ in our case. However, in those examples of non-linear sigma model effective theories, the coefficients of the higher-derivative terms are undetermined and they have to be obtained phenomenologically since the underlying theory cannot be solved (QCD) or is unknown (symmetry-breaking sector). However, in the case considered here, these coefficients are explicitly obtained in terms of the brane tension $f$ from the underlying theory, which is given by the Nambu-Goto-like brane action.

\section{Kaluza-Klein gauge bosons and the Higgs mechanism}

As commented in the introduction, the isometries in the $B$ space are considered as gauge transformation in the Kaluza-Klein theories [8]. In this section we study under which circumstances the GB associated to the isometry breaking can give rise to the longitudinal components of the Kaluza-Klein gauge bosons, as in the standard Higgs mechanism.

We start with the Hilbert-Einstein action for the gravitational field in $D$ dimensions plus the brane action: $S=S_{G}+S_{B}$, i.e.,

$$
S=\frac{-1}{16 \pi G_{D}} \int_{M_{D}} d^{D} z \sqrt{G} R_{D}-\frac{f^{4}}{4} \int_{M_{4}} d^{4} x \sqrt{g} G_{M N} g^{\mu \nu} \partial_{\mu} Y^{M} \partial_{\nu} Y^{N},
$$

where $z=(x, y)$ are the coordinates defined on $M_{D}, x$ and $y$ being the coordinates defined on $M_{4}$ and $B$, respectively, and $R_{D}$ is the $D$-dimensional scalar curvature. For the particular case of an $S^{1}$ compactified extra dimension (Abelian case), $M_{D}=M_{4} \times S^{1}$ and $G(B)=U(1)$. As usually done in the Kaluza-Klein approach, we consider the metric ansatz

$$
G_{M N}=\left(\begin{array}{cc}
\tilde{g}_{\mu \nu}(x)-B_{\mu}(x) B_{v}(x) & B_{\mu}(x) \\
B_{v}(x) & -1
\end{array}\right) .
$$

According to the Kaluza-Klein philosophy, the translations on the compactified fifth dimension $y \rightarrow y^{\prime}=y+R \theta(x)$, where $R$ is the compactification radius, can be understood as standard gauge $U(1)$ transformations. With the above metric, the five-dimensional gravitational action contains the terms

$$
S_{G}=\frac{-1}{16 \pi G_{N}} \int d^{4} x \sqrt{\tilde{g}} \widetilde{R}-\frac{1}{4} \int d^{4} x \sqrt{\tilde{g}} F_{\mu \nu} F^{\mu \nu},
$$

where $\widetilde{R}$ is the scalar curvature corresponding to the $\tilde{g}_{\mu \nu}$ metric, $G_{5}$ and $G_{N}=1 / M_{P}^{2}$ are related by $G_{5}=2 \pi R G_{N}, F_{\mu \nu}=\partial_{\mu} A_{\nu}-\partial_{\nu} A_{\mu}$ and $B_{\mu}=k A_{\mu}$. On the other hand, the induced metric $g_{\mu \nu}$ turns out, in this case, to be

$$
g_{\mu \nu}=\tilde{g}_{\mu \nu}-D_{\mu} Y D_{\nu} Y
$$

and 


$$
\sqrt{g}=\sqrt{\tilde{g}}\left(1-\frac{1}{2} \tilde{g}^{\mu \nu} D_{\mu} Y D_{\nu} Y+\cdots\right),
$$

where the covariant derivative is defined as

$$
D_{\mu} Y=\partial_{\mu} Y-B_{\mu}=\frac{1}{f^{2}}\left(\partial_{\mu} \pi-k f^{2} A_{\mu}\right)=\frac{1}{f^{2}} D_{\mu} \pi .
$$

Thus the total low-energy action can be written as

$$
\begin{aligned}
S= & \frac{-1}{16 \pi G_{N}} \int d^{4} x \sqrt{\tilde{g}} \widetilde{R}-\frac{1}{4} \int d^{4} x \sqrt{\tilde{g}} F_{\mu \nu} F^{\mu \nu} \\
& -f^{4} \int_{M_{4}} d^{4} x \sqrt{\tilde{g}}+\frac{f^{4}}{2} \int_{M_{4}} d^{4} x \sqrt{\tilde{g}} \tilde{g}^{\mu \nu} D_{\mu} Y D_{\nu} Y .
\end{aligned}
$$

As a consequence, the gauge bosons (graviphotons) get a mass term

$$
\frac{k^{2} f^{4}}{2} \int d^{4} x \sqrt{\tilde{g}} A_{\mu} A^{\mu}
$$

i.e., the gauge boson mass is $M^{2}=k^{2} f^{4}=16 \pi f^{4} / M_{P}^{2}$, which is typically very small. For example, in order to be able to generate the $W$ mass through this Higgs mechanism, the brane tension should be as large as the geometrical average between the electroweak and the Planck scale. More precisely,

$$
f^{2}=\frac{M_{W} M_{P}}{4 \sqrt{\pi}} .
$$

In the general non-Abelian case, we consider again the brane and space-time structure $M_{D}=M_{4} \times B$. As usual in the Kaluza-Klein approach the metric ansatz is taken to be:

$$
G_{M N}=\left(\begin{array}{cc}
\tilde{g}_{\mu \nu}(x)-g_{m n}^{\prime}(y) B_{\mu}^{m}(x, y) B_{v}^{n}(x, y) & B_{\mu}^{n}(x, y) \\
B_{v}^{m}(x, y) & -g_{m n}^{\prime}(y)
\end{array}\right),
$$

where $B_{\mu}^{n}(x, y)=\xi_{a}^{n}(y) A_{\mu}^{a}(x)$ with $\xi_{a}^{n}(y)$ the Killing vectors corresponding to the isometry group $G(B)$ introduced above. Now the gauge transformations are $y^{m} \rightarrow y^{\prime m}=$ $y^{m}+\xi_{a}^{m}(y) \epsilon^{a}(x)$. As is well known in this case, the gravitational action $S_{G}$ can be written as

$$
S_{G}=\frac{-1}{16 \pi G_{N}} \int d^{4} x \sqrt{\tilde{g}} \widetilde{R}-\frac{\left\langle\xi_{a}^{n} \xi_{b}^{m} g_{m n}^{\prime}\right\rangle}{16 \pi G_{N}} \frac{1}{4} \int_{M_{4}} d^{4} x \sqrt{\tilde{g}} F_{\mu \nu}^{a} F^{\mu \nu b},
$$

where $F_{\mu \nu}^{a}=\partial_{\mu} A_{\nu}^{a}-\partial_{\nu} A_{\mu}^{a}-C_{a b c} A_{\mu}^{b} A_{\nu}^{c}$ and

$$
16 \pi G_{N}=\frac{16 \pi G_{D}}{\int_{B} d^{D-4} y \sqrt{g^{\prime}}} .
$$

In order to obtain the standard Yang-Mills action, the normalization of the Killing vectors is given by

$$
\left\langle\xi_{a}^{m}(y) \xi_{b}^{n}(y) g_{m n}^{\prime}(y)\right\rangle=k^{2} \delta_{a b},
$$


where $g_{m n}^{\prime}(y)$ is the $B$ space-time metric, again $k^{2}=16 \pi G_{N}$ and the brackets are defined as the $B$ manifold average [17]

$$
\langle f(y)\rangle=\frac{\int_{B} d^{D-4} y \sqrt{g^{\prime}} f(y)}{\int_{B} d^{D-4} y \sqrt{g^{\prime}}} .
$$

The induced metric is

$$
g_{\mu \nu}=\tilde{g}_{\mu \nu}-\Delta_{\mu} Y^{m} \Delta_{\nu} Y^{n} g_{m n}^{\prime}
$$

and

$$
\sqrt{g}=\sqrt{\tilde{g}}\left(1-\frac{1}{2} \tilde{g}^{\mu \nu} \Delta_{\mu} Y^{m} \Delta_{\nu} Y^{n} g_{m n}^{\prime}+\cdots\right),
$$

where the covariant derivative is defined as

$$
\Delta_{\mu} Y^{m}=\partial_{\mu} Y^{m}-\xi_{a}^{m} A_{\mu}^{a},
$$

which can be written as

$$
\begin{aligned}
\Delta_{\mu} Y^{m} & =\frac{\partial Y^{m}}{\partial \pi^{\alpha}} \partial_{\mu} \pi^{\alpha}-\xi_{a}^{m} A_{\mu}^{a} \\
& =\frac{1}{k f^{2}} \xi_{\alpha}^{m}\left(Y_{0}\right)\left(\partial_{\mu} \pi^{\alpha}-k f^{2} A_{\mu}^{\alpha}\right)-\xi_{i}^{m}\left(Y_{0}\right) A_{\mu}^{i}+\mathcal{O}\left(\pi^{2}\right) .
\end{aligned}
$$

Since the $i$ indices correspond to the generators of the isotropy group $H\left(Y_{0}\right)$, the Killing fields vanish at $Y_{0}$, i.e., $\xi_{i}^{m}\left(Y_{0}\right)=0$ and the last term vanishes.

Therefore the brane action $S_{B}$ is

$$
S_{B}=-f^{4} \int_{M_{4}} d^{4} x \sqrt{\tilde{g}}+\frac{1}{2} \int_{M_{4}} d^{4} x \sqrt{\tilde{g}} \tilde{g}^{\mu \nu} h_{\alpha \beta} D_{\mu} \pi^{\alpha} D_{\nu} \pi^{\beta}+\mathcal{O}\left(\pi^{4}\right) .
$$

where $D_{\mu} \pi^{\alpha}=\partial_{\mu} \pi^{\alpha}-k f^{2} A_{\mu}^{\alpha}$. Thus the gauge boson mass matrix is

$$
M_{\alpha \beta}^{2}=k^{2} f^{4} h_{\alpha \beta}(0) .
$$

Remember that $Y^{m}(x)=Y_{0}^{m}$ corresponds to $\pi^{\alpha}=0$. As commented on before, not all the the gauge bosons will acquire a mass by this mechanism. Only those associated to the broken $X_{\alpha}$ generators will, their number being determined by the dimension of the $K=$ $G / H$ space. In any case, two important comments are in order. First, as it happened in the Abelian case, the gauge boson masses are quite small whenever $f \ll M_{P}$. On the other hand it should be remembered that in the standard KK picture, having gauge couplings $g$ small enough to have a sensible weak coupling limit (say $g<1$ ) requires having extra dimensions of a typical size of the order of the Planck length, since $g^{2}$ is of the order of $k^{2} / R^{2}$ (see, for instance, [8]). Thus, for the interesting case of large extra dimensions and $f \ll M_{D}$, graviphotons can be considered massless and decoupled from the rest of the low-energy particles. In this case we can safely assume that the Higgs mechanism has not taken place and the GB can be considered as the only relevant low-energy new degrees of freedom. 


\section{Couplings to the Standard Model fields}

As we have shown in the previous sections, the induced metric on the brane depends on both the four-dimensional bulk metric components $\tilde{g}_{\mu \nu}$ and the Goldstone bosons $\pi^{\alpha}$. In the following, we will assume that the physical space-time metric is $\tilde{g}_{\mu \nu}$, whereas the contribution of the GB to the brane metric can be detected only through their couplings to the Standard Model fields. In order to obtain such couplings, we start from the Sundrum effective action for the SM fields [5], which is basically the SM action defined on a curved space-time $M_{4}$ whose metric is the induced metric $g_{\mu \nu}$. Let us give the results for the different kinds of fields (we will follow the notation in [16]):

\subsection{Scalars}

For a scalar field we start from the action

$$
S_{\Phi}=\frac{1}{2} \int_{M_{4}} d^{4} x \sqrt{g} g^{\mu \nu} \partial_{\mu} \Phi \partial_{\nu} \Phi,
$$

which can be written up to $\mathcal{O}\left(p^{2}\right)$ as

$$
\begin{aligned}
S_{\Phi}^{(2)}= & \frac{1}{2} \int_{M_{4}} d^{4} x \sqrt{\tilde{g}} \tilde{g}^{\mu \nu} \partial_{\mu} \Phi \partial_{\nu} \Phi \\
& +\frac{1}{2 f^{4}} \int_{M_{4}} d^{4} x \sqrt{\tilde{g}} h_{\alpha \beta}(\pi) \partial_{\mu} \Phi \partial_{\nu} \Phi \partial^{\mu} \pi^{\alpha} \partial^{\nu} \pi^{\beta} \\
& -\frac{1}{4 f^{4}} \int_{M_{4}} d^{4} x \sqrt{\tilde{g}} \tilde{g}^{\mu \nu} \partial_{\mu} \Phi \partial_{\nu} \Phi \tilde{g}^{\rho \sigma} h_{\alpha \beta}(\pi) \partial_{\rho} \pi^{\alpha} \partial_{\sigma} \pi^{\beta} .
\end{aligned}
$$

\subsection{Fermions}

In order to introduce fermions on the brane, we will first extend the vielbein formalism to $M_{4} \times B$. For this purpose we give the necessary notation for the different kinds of indices. We will use $A, B, \ldots=0, \ldots, D-1$ to denote flat indices in the bulk, $M, N, \ldots=$ $0, \ldots, D-1$ for curved indices in the bulk, $i, j, \ldots=0, \ldots, 3$ flat indices in $M_{4}$ (not to be confused with the gauge indices for $H$ introduced in Section 2$), \mu, v, \ldots=0, \ldots, 3$ curved indices in $M_{4}, \bar{m}, \bar{n}, \ldots=4, \ldots, D-1$ flat indices in $B$ and $m, n, \ldots=4, \ldots, D-1$ curved indices in $B$. Let us consider the following ansatz for the vielbein in $M_{4} \times B$ :

$$
E_{M}^{A}=\left(\begin{array}{cc}
\tilde{e}_{\mu}^{i} & B_{\mu}^{\bar{m}} \\
0 & e_{m}^{\bar{m}}
\end{array}\right),
$$

and the inverse vielbein:

$$
E_{A}^{M}=\left(\begin{array}{cc}
\tilde{e}_{i}^{\mu} & -B_{i}^{m} \\
0 & e_{\bar{m}}^{m}
\end{array}\right) .
$$

With these definitions we have: $G_{M N}=\eta_{A B} E_{M}^{A} E_{N}^{B}, \tilde{g}_{\mu \nu}=\eta_{i j} \tilde{e}_{\mu}^{i} \tilde{e}_{\nu}^{j}$ and $g_{m n}=\eta_{\bar{m} \bar{n}} e_{m}^{\bar{m}} e_{n}^{\bar{n}}$, where $g_{m n}=-g_{m n}^{\prime}$ and $\eta_{\bar{m} \bar{n}}=-\delta_{\bar{m} \bar{n}}$. 
In order to obtain the induced vierbein on the brane $e_{\mu}^{i}$, we follow Sundrum [5] in defining:

$$
e_{\mu}^{i}=R_{A}^{i} E_{M}^{A} \partial_{\mu} Y^{M}
$$

where $R_{B}^{A}$ are the components of a Lorentz transformation in the $D$-dimensional tangent space given by:

$$
R(x)=\exp \left(i \theta_{i \bar{m}}(x) J^{(i \bar{m})}\right),
$$

with $\theta_{i \bar{m}}(x)$ the transformation parameters and $J^{(A B)}$ the generators of Lorentz transformations in $D$ dimensions. In particular, they can be written as:

$$
J_{D}^{(A B) C}=i \eta^{C E}\left(\delta_{E}^{A} \delta_{D}^{B}-\delta_{D}^{A} \delta_{E}^{B}\right) .
$$

We will only consider those transformations $J^{(i \bar{m})}$ mixing internal and external indices. Let us take those tangent vectors given by $E_{M}^{A}(Y) \partial_{\mu} Y^{M}$, and act on them with the Lorentz transformation given in (45). If we impose that the transformed vectors be orthogonal to the $\bar{m}$ directions, i.e., they only have non-vanishing four-dimensional $i$ components, they will then satisfy:

$$
R_{A}^{\bar{m}} E_{M}^{A}(Y) \partial_{\mu} Y^{M}=0 .
$$

If $R_{A}^{\bar{m}}$ satisfies this condition, the induced vierbein defined in (44) then possesses the properties of a vierbein, i.e., $e_{\mu}^{i} e_{\nu}^{j} \eta_{i j}=g_{\mu \nu}$, where $g_{\mu \nu}$ is the induced metric on the brane given in (1). This definition allows us to introduce chiral fermions in the brane in a straightforward way, as we will show below.

Using the expression for the vielbein given in (44), we can find:

$$
e_{\mu}^{i}=R_{j}^{i} \tilde{e}_{\mu}^{j}+R_{\bar{p}}^{i} e_{m}^{\bar{p}}\left(\partial_{\mu} Y^{m}+B_{\mu}^{m}\right) .
$$

The expressions for $R_{A}^{i}$ are determined from Eq. (47). In particular, since we are mainly interested in the couplings of the Goldstone bosons to fermions to lowest order, it will be sufficient to calculate the components $R_{A}^{i}$ to first (second) order in $\theta_{i \bar{m}}(x)$. Thus, in particular, we will need:

$$
\begin{aligned}
R_{j}^{i} & =\delta_{j}^{i}-\frac{1}{2} \theta_{k \bar{p}} \theta_{j \bar{q}} \eta^{\bar{p} \bar{q}} \eta^{i k}, \\
R_{\bar{p}}^{i} & =-\theta_{j \bar{p}} \eta^{i j}, \quad R_{i}^{\bar{q}}=\theta_{i \bar{p}} \eta^{\bar{p} \bar{q}}, \\
R_{\bar{m}}^{\bar{q}} & =\delta_{\bar{m}}^{\bar{q}}-\frac{1}{2} \theta_{j \bar{p}} \theta_{k \bar{m}} \eta^{\bar{p} \bar{q}} \eta^{j k} .
\end{aligned}
$$

From (47) we get:

$$
\theta_{i \bar{q}}=-\tilde{e}_{i}^{\mu}\left(B_{\bar{q} \mu}+\eta_{\bar{q} \bar{m}} e_{m}^{\bar{m}} \partial_{\mu} Y^{m}\right) .
$$

Using this result and the definition of the induced vierbein in (44), we obtain, up to second order in the gauge fields and the derivatives of the coordinates:

$$
e_{\mu}^{i}=\tilde{e}_{\mu}^{i}+\frac{1}{2} T_{\mu}^{i}, \quad e_{i}^{\mu}=\tilde{e}_{i}^{\mu}-\frac{1}{2} T_{i}^{\mu},
$$

where 


$$
\begin{aligned}
T_{\mu \nu} & =-g_{m n}^{\prime}\left(\partial_{\mu} Y^{m}+B_{\mu}^{m}\right)\left(\partial_{\nu} Y^{n}+B_{\nu}^{n}\right), \\
T_{\mu}^{i} & =\tilde{e}_{\nu}^{i} \tilde{g}^{\nu \rho} T_{\mu \rho},
\end{aligned}
$$

with these definitions the induced metric can be written as $g_{\mu \nu}=\tilde{g}_{\mu \nu}+T_{\mu \nu}$.

We also need to know the expression of the induced spin connection, defined as:

$$
\omega_{\mu}^{i j}=e_{\nu}^{i} \eta^{j k}\left(\partial_{\mu} e_{k}^{v}+e_{k}^{\lambda} \Gamma_{\mu \lambda}^{\nu}\right),
$$

where the induced Christoffel symbols on the brane are given by:

$$
\Gamma_{\mu \lambda}^{\nu}=\widetilde{\Gamma}_{\mu \lambda}^{\nu}-T^{\nu \rho} \tilde{g}_{\rho \sigma} \widetilde{\Gamma}_{\mu \lambda}^{\sigma}+\frac{1}{2} \tilde{g}^{\nu \rho}\left(\partial_{\mu} T_{\rho \lambda}+\partial_{\lambda} T_{\rho \mu}-\partial_{\rho} T_{\mu \lambda}\right) .
$$

With these expressions we can obtain the form of the induced spin connection in terms of the spin connection associated to the metric $\tilde{g}_{\mu \nu}$. We have, up to first order in $T$ :

$$
\omega_{\mu}^{i j}=\tilde{\omega}_{\mu}^{i j}+\frac{1}{2} \tilde{e}_{\nu}^{i} \tilde{e}_{k}^{\lambda} \eta^{j k} \tilde{g}^{\nu \rho}\left(T_{\rho \mu ; \lambda}-T_{\mu \lambda ; \rho}\right) .
$$

The Dirac action for a massless fermion on the brane can be written as:

$$
S=\int d^{4} x \sqrt{g} i \bar{\psi} e_{i}^{\mu} \gamma^{i}\left(\partial_{\mu}+\Omega_{\mu}\right) \psi
$$

where $\Omega_{\mu}=\frac{1}{8} \omega_{\mu}^{i j}\left[\gamma_{i}, \gamma_{j}\right]$ and $\left\{\gamma^{i}, \gamma^{j}\right\}=2 \eta^{i j}$. Using the above equations we can expand the Dirac action in terms of the metric $\tilde{g}_{\mu \nu}$ and the $T_{\mu \nu}$ tensors defined before. We get, up to first order in $T$ :

$$
\begin{aligned}
S= & \int d^{4} x \sqrt{\tilde{g}} i \bar{\psi} \tilde{e}_{i}^{\mu} \gamma^{i}\left(\partial_{\mu}+\widetilde{\Omega}_{\mu}\right) \psi+\frac{1}{2} \int d^{4} x \sqrt{\tilde{g}} i T_{\mu}^{\mu} \bar{\psi} \tilde{e}_{i}^{\mu} \gamma^{i}\left(\partial_{\mu}+\widetilde{\Omega}_{\mu}\right) \psi \\
& -\frac{1}{2} \int d^{4} x \sqrt{\tilde{g}} i \bar{\psi} T_{i}^{\mu} \gamma^{i}\left(\partial_{\mu}+\widetilde{\Omega}_{\mu}\right) \psi \\
& +\frac{1}{4} \int d^{4} x \sqrt{\tilde{g}} i \bar{\psi} \tilde{e}_{i}^{\mu} \gamma^{i}\left(T_{v ; \mu}^{v}-T_{\mu ; \nu}^{v}\right) \psi
\end{aligned}
$$

In the simplest case, in which the Kaluza-Klein gauge bosons are absent, we can expand the Dirac action in powers of the properly normalized Goldstone bosons $\pi^{\alpha}$. Up to $\mathcal{O}\left(p^{2}\right)$, we obtain:

$$
\begin{aligned}
S= & \int d^{4} x \sqrt{\tilde{g}} i \bar{\psi} \tilde{e}_{i}^{\mu} \gamma^{i}\left(\partial_{\mu}+\tilde{\Omega}_{\mu}\right) \psi \\
& -\frac{i}{2 f^{4}} \int d^{4} x \sqrt{\tilde{g}} h_{\alpha \beta}(\pi) \tilde{g}^{\mu \nu} \partial_{\mu} \pi^{\alpha} \partial_{\nu} \pi^{\beta} \bar{\psi} \tilde{e}_{i}^{\rho} \gamma^{i}\left(\partial_{\rho}+\widetilde{\Omega}_{\rho}\right) \psi \\
& +\frac{i}{2 f^{4}} \int d^{4} x \sqrt{\tilde{g}} \bar{\psi} h_{\alpha \beta}(\pi) \tilde{g}^{\mu \nu} \partial_{\mu} \pi^{\alpha} \not \partial \pi^{\beta}\left(\partial_{\nu}+\widetilde{\Omega}_{\nu}\right) \psi \\
& -\frac{i}{4 f^{4}} \int d^{4} x \sqrt{\tilde{g}} \bar{\psi} h_{\alpha \beta}(\pi) \tilde{g}^{\mu \nu}\left(\not \partial\left(\partial_{\mu} \pi^{\alpha} \partial_{\nu} \pi^{\beta}\right)-\partial_{\mu}\left(\not \partial \pi^{\alpha} \partial_{\nu} \pi^{\beta}\right)\right) \psi .
\end{aligned}
$$

In particular, this way of introducing the couplings of Goldstone bosons to fermions allows us to consider chiral fermions in a straightforward way. Thus for the fermionic sector of the Standard Model we get: 


$$
\begin{gathered}
S=i \int d^{4} x \sqrt{\tilde{g}}\left(\overline{\mathcal{Q}} \not D^{Q} \mathcal{Q}+\overline{\mathcal{L}} \not D^{L} \mathcal{L}\right) \\
-\frac{i}{2 f^{4}} \int d^{4} x \sqrt{\tilde{g}} h_{\alpha \beta}(\pi) \tilde{g}^{\mu \nu} \partial_{\mu} \pi^{\alpha} \partial_{\nu} \pi^{\beta}\left(\overline{\mathcal{Q}} \not D^{Q} \mathcal{Q}+\overline{\mathcal{L}} \not D^{L} \mathcal{L}\right) \\
+\frac{i}{2 f^{4}} \int d^{4} x \sqrt{\tilde{g}} h_{\alpha \beta}(\pi) \tilde{g}^{\mu \nu}\left(\overline{\mathcal{Q}} \partial_{\mu} \pi^{\alpha} \not \partial \pi^{\beta} D_{\nu}^{Q} \mathcal{Q}+\overline{\mathcal{L}} \partial_{\mu} \pi^{\alpha} \not \partial \pi^{\beta} D_{\nu}^{L} \mathcal{L}\right) \\
-\frac{i}{4 f^{4}} \int d^{4} x \sqrt{\tilde{g}} h_{\alpha \beta}(\pi) \tilde{g}^{\mu \nu}\left[\overline{\mathcal{Q}}\left(\not \partial\left(\partial_{\mu} \pi^{\alpha} \partial_{\nu} \pi^{\beta}\right)-\partial_{\mu}\left(\not \partial \pi^{\alpha} \partial_{\nu} \pi^{\beta}\right)\right) \mathcal{Q}\right. \\
\left.+\overline{\mathcal{L}}\left(\not \partial\left(\partial_{\mu} \pi^{\alpha} \partial_{\nu} \pi^{\beta}\right)-\partial_{\mu}\left(\not \partial \pi^{\alpha} \partial_{\nu} \pi^{\beta}\right)\right) \mathcal{L}\right],
\end{gathered}
$$

where we have:

$$
\begin{aligned}
& \not D^{Q}=\tilde{e}_{i}^{\mu} \gamma^{i} D_{\mu}^{Q}=\tilde{e}_{i}^{\mu} \gamma^{i}\left(\partial_{\mu}+\widetilde{\Omega}_{\mu}+G_{\mu}+W_{\mu} P_{L}+i g^{\prime}\left(Y_{L}^{Q} P_{L}+Y_{R}^{Q} P_{R}\right) B_{\mu}\right) \\
& \not D^{L}=\tilde{e}_{i}^{\mu} \gamma^{i} D_{\mu}^{L}=\tilde{e}_{i}^{\mu} \gamma^{i}\left(\partial_{\mu}+\widetilde{\Omega}_{\mu}+W_{\mu} P_{L}+i g^{\prime}\left(Y_{L}^{L} P_{L}+Y_{R}^{L} P_{R}\right) B_{\mu}\right),
\end{aligned}
$$

and $Y_{L, R}^{Q, L}$ denote the hypercharge matrices for left or right quarks and leptons. The left and right projectors $P_{L, R}$ are defined as usual from the four-dimensional $\gamma_{5}$ matrix. The Yukawa sector can be obtained in a straightforward way, and we obtain:

$$
\begin{aligned}
S_{\mathrm{YK}}=-\int d^{4} x & \sqrt{\tilde{g}}\left(1-\frac{1}{2 f^{4}} h_{\alpha \beta}(\pi) \tilde{g}^{\mu \nu} \partial_{\mu} \pi^{\alpha} \partial_{\nu} \pi^{\beta}\right) \\
& \times\left(\overline{\mathcal{Q}}_{L} \Phi H_{Q} \mathcal{Q}_{R}+\overline{\mathcal{L}}_{L} \Phi H_{L} \mathcal{L}\right)+\text { h.c. }
\end{aligned}
$$

Here $H_{Q, L}$ denote the Yukawa matrices and $\Phi$ is the Higgs doublet.

\subsection{Gauge bosons}

For the Yang-Mills action on the brane we can follow similar steps, and we find:

$$
\begin{aligned}
S_{\mathrm{YM}}= & \frac{\operatorname{tr}}{2 g^{2}} \int d^{4} x \sqrt{g} g^{\mu \rho} g^{\nu \sigma} G_{\mu \nu} G_{\rho \sigma} \\
= & \frac{\operatorname{tr}}{2 g^{2}} \int d^{4} x \sqrt{\tilde{g}} \tilde{g}^{\mu \rho} \tilde{g}^{\nu \sigma} G_{\mu \nu} G_{\rho \sigma}+\frac{\operatorname{tr}}{4 g^{2}} \int d^{4} x \sqrt{\tilde{g}} T_{\mu}^{\mu} \tilde{g}^{\mu \rho} \tilde{g}^{\nu \sigma} G_{\mu \nu} G_{\rho \sigma} \\
& -\frac{\operatorname{tr}}{g^{2}} \int d^{4} x \sqrt{\tilde{g}} T^{\mu \rho} \tilde{g}^{\nu \sigma} G_{\mu \nu} G_{\rho \sigma},
\end{aligned}
$$

where $T_{\mu \nu}$ is defined in (52). Finally expanding the $T$ terms in Goldstone bosons, we get:

$$
\begin{aligned}
S_{\mathrm{YM}}= & \frac{\operatorname{tr}}{2 g^{2}} \int d^{4} x \sqrt{\tilde{g}} \tilde{g}^{\mu \rho} \tilde{g}^{\nu \sigma} G_{\mu \nu} G_{\rho \sigma} \\
& -\frac{\operatorname{tr}}{4 g^{2} f^{4}} \int d^{4} x \sqrt{\tilde{g}} \tilde{g}^{\mu \nu} h_{\alpha \beta}(\pi)\left(\partial_{\mu} \pi^{\alpha} \partial_{\nu} \pi^{\beta}\right) \tilde{g}^{\mu \rho} \tilde{g}^{\nu \sigma} G_{\mu \nu} G_{\rho \sigma} \\
& +\frac{\operatorname{tr}}{g^{2} f^{4}} \int d^{4} x \sqrt{\tilde{g}} h_{\alpha \beta}(\pi)\left(\partial_{\lambda} \pi^{\alpha} \partial_{\kappa} \pi^{\beta}\right) \tilde{g}^{\mu \lambda} \tilde{g}^{\rho \kappa} \tilde{g}^{\nu \sigma} G_{\mu \nu} G_{\rho \sigma} .
\end{aligned}
$$

From the above discussion we see that the GB always interact by pairs with the SM matter. In addition, due to their geometric origin, those interactions are very similar to the gravitational interactions since the $\pi$ fields couple to all the matter fields with the same 
strength, which is suppressed by a factor $f^{4}$. This is quite interesting since it could explain why they have not been observed so far, provided they exist at all. However, moderate values of the brane tension around the $\mathrm{TeV}$ scale could make their production possible in the next generation of colliders.

\section{Conclusions}

In this work we have studied the effective action describing the low-energy dynamics of the GB, which appear when the higher-dimensional space-time manifold isometry group is spontaneously broken by the presence of a three-brane Universe. From the $(3+1)$ dimensional point of view, those GB can be considered as some kind of new scalar fields whose dynamics is given by the non-linear sigma model Lagrangian corresponding to the coset manifold $K=G / H$. Eventually, the GB can also get some mass terms due to small deviations from the simple ideal exact isometry pattern.

This spontaneous symmetry breaking gives rise, through the Higgs mechanism, to a mass matrix for the KK graviphotons associated to the isometries of the compactified space $B$. However, for the interesting case of large extra dimensions and $f \ll M_{D}$, the graviphotons decouple from the low-energy theory and their masses become very small. We can thus consider the GB as the only relevant degrees of freedom on the brane in the low-energy regime.

In order to make further studies of the possible phenomenological implications of those GB brane excitations, we have considered their corresponding couplings with the SM particles including scalars, fermions (chiral and non-chiral) and gauge bosons.

In the above-mentioned scenario of large extra dimensions with $f \ll M_{D}$, for appropriate values of $f$ and the GB masses, these scalar particles are the only brane states that could be probed in the next generation of colliders such as the LHC. In this case, the effective couplings obtained here provide the necessary tools to compute the cross sections and the expected rates of new exotic events in terms of $f$ and the GB masses only. Work is in progress in that direction.

\section{Acknowledgements}

This work has been partially supported by the Ministerio de Educación y Ciencia (Spain) (CICYT AEN 97-1693 and PB98-0782).

\section{References}

[1] N. Arkani-Hamed, S. Dimopoulos, G. Dvali, Phys. Lett. B 429 (1998) 263.

[2] N. Arkani-Hamed, S. Dimopoulos, G. Dvali, Phys. Rev. D 59 (1999) 086004.

[3] I. Antoniadis, N. Arkani-Hamed, S. Dimopoulos, G. Dvali, Phys. Lett. B 436 (1998) 257.

[4] T. Banks, M. Dine, A. Nelson, JHEP 9906 (1999) 014.

[5] R. Sundrum, Phys. Rev. D 59 (1999) 085009. 
[6] T. Kaluza, Sitzungsberichte of the Prussian Acad. Sci. 966 (1921).

[7] O. Klein, Z. Phys. 37 (1926) 895.

[8] D. Bailin, A. Love, Rep. Prog. Phys. 50 (1987) 1087.

[9] G. Giudice, R. Rattazzi, J.D. Wells, Nucl. Phys. B 544 (1999) 3.

[10] E.A. Mirabelli, M. Perelstein, M.E. Peskin, Phys. Rev. Lett. 82 (1999) 2236.

[11] M. Bando, T. Kugo, T. Noguchi, K. Yoshioka, Phys. Rev. Lett. 83 (1999) 3601.

[12] J. Hisano, N. Okada, Phys. Rev. D 61 (2000) 106003.

[13] T. Kugo, K. Yoshioka, hep-ph/9912496.

[14] P.A.M. Dirac, Proc. R. Soc. London A 268 (1962) 57.

[15] S. Helgason, Differential Geometry, Lie Groups, and Symmetric Spaces, Academic Press, New York, 1978.

[16] A. Dobado, A. Gómez-Nicola, A.L. Maroto, J.R. Peláez, Effective Lagrangians for the Standard Model, Springer-Verlag, Heidelberg, 1997.

[17] S. Weinberg, Phys. Lett. B 125 (1983) 265. 